\title{
Random two-frame phase-shifting interferometry via minimization of coefficient of variation
}

Cite as: Appl. Phys. Lett. 115, 121107 (2019); https://doi.org/10.1063/1.5118296

Submitted: 03 July 2019. Accepted: 22 August 2019. Published Online: 19 September 2019

Zhongtao Cheng (D), Dong Liu, and Lei Zhang
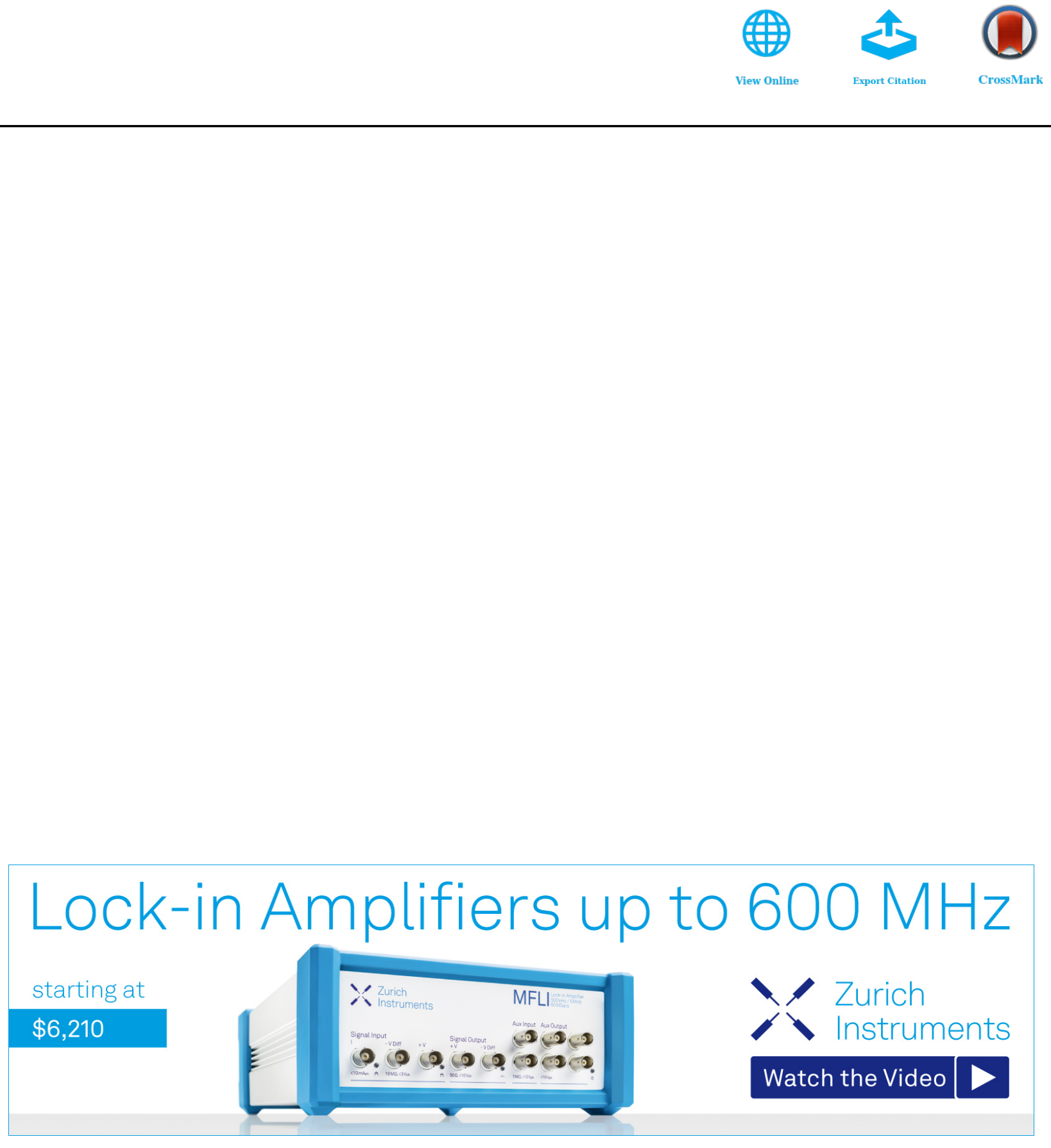


\title{
Random two-frame phase-shifting interferometry via minimization of coefficient of variation
}

\author{
Cite as: Appl. Phys. Lett. 115, 121107 (2019); doi: 10.1063/1.5118296 \\ Submitted: 3 July 2019 - Accepted: 22 August 2019 . \\ Published Online: 19 September 2019
}

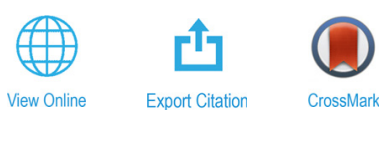

\author{
Zhongtao Cheng, ${ }^{1, \text { a) }}$ (D) Dong Liu, ${ }^{2}$ and Lei Zhang ${ }^{3}$
}

\begin{abstract}
AFFILIATIONS
'Division of Engineering and Applied Science, California Institute of Technology, 1200 E. California Blvd., Pasadena, California 91125, USA

${ }^{2}$ State Key Laboratory of Modern Optical Instrumentation, College of Optical Science and Engineering, Zhejiang University, Hangzhou, Zhejiang 310027, China

${ }^{3}$ Key Laboratory of Opto-Electronic Information Acquisition and Manipulation Ministry of Education, Anhui University, Hefei 230601, China
\end{abstract}

${ }^{a}$ Author to whom correspondence should be addressed: Zhongtao.Cheng@caltech.edu

\begin{abstract}
Random two-frame phase-shifting interferometry (PSI) is an advanced technique to retrieve the phase information from as few as two interferograms with unknown phase steps. Because of the advantages of no requirement for accurate phase shifters and much less time for data acquisition and processing, random two-frame PSI is attracting more and more interest in fast and high-precision optical metrology. However, reconstructing the phase from only two interferograms is challenging because it is an ill-posed problem in essence, especially when the phase step is unknown. Although some solutions have been proposed for this problem to date, most of them require complicated preprocessing or special usage preconditions for interferograms to be demodulated. In this letter, we developed an elegant phase reconstruction method for random two-frame PSI, which is much different from frameworks of existing methods. In the proposed approach, the phase of random two-frame PSI can be accurately reconstructed using the phase step value which minimizes the coefficient of variation (CV) of the modulation term of interferograms. Sufficient numerical simulations and experimental data demonstrate the high accuracy and high efficiency of this CV minimization (CVM) method. Moreover, its performance is not limited by the number of fringes in interferograms, in contrast to existing state-of-the-art approaches. We anticipate extensive applications of the CVM method in random two-frame PSI in the future.
\end{abstract}

Published under license by AIP Publishing. https://doi.org/10.1063/1.5118296

As an advanced technique for high-precision optical metrology, phase-shifting interferometry (PSI) plays important roles in optical shop testing, ${ }^{1}$ laser wavefront diagnosis, ${ }^{2}$ and threedimensional shape measurement, ${ }^{3-5}$ among others. In standard PSI, multiple interferograms with known phase steps (typically, e.g., $\pi / 2 \mathrm{rad}$ ) are required to extract the underlying phase related to the physical quantities to be measured. ${ }^{1,5-7}$ In such approaches, the accuracy of the phase reconstruction is greatly limited by the accuracy of the phase shifter because the phase steps between interferograms must match the designated ones. On the other hand, the need for capturing many interferograms increases the possible detrimental effects from the mechanical vibrations and air turbulence on measurements and is not acceptable in applications related to high-speed transient events. In theory, a single-frame interferogram can be used to demodulate the phase information without the above limitations, known as single-frame interferometry. ${ }^{8-10}$ However, the single-frame interferometry only reduces the recording time for interferometric measurement but at the cost of much more computational load for the phase extraction. Moreover, it may be susceptible to sign ambiguity and obtain erroneous results when demodulating closed fringes. By adding another phase-shifting interferogram on the basis of single-frame interferometry, random two-frame PSI is attracting more and more interest in recent years because it is found to be a very good compromise between the capture time and phase reconstruction accuracy. That is, two frames are the minimal number of interferograms required for phase demodulation without sign ambiguity. The phase step between the two frames can be random and unknown (except singular 0 and $\pi$ $\mathrm{rad}$ ), which also alleviates the strict requirement for accurate phase shifters. 
However, reconstructing the phase from only two interferograms is also challenging because it is an ill-posed problem in essence, especially when the phase step is unknown. Some solutions have been proposed for this problem to date. In Ref. 11, the authors employ the regularized optical flow (OF) algorithm in the field of computer vision to obtain the fringe direction map and then perform the spiral phase transform to one of the interferograms to determine its quadrature signal. The phase map is extracted from the original and quadrature signals subsequently. Another method to obtain the quadrature signal is to consider the two-frame interferograms as independent vectors and make two mutually orthogonal vectors through the standard Gram-Schmidt (GS) orthonormalization process, assuming that there are more than one fringe in the interferograms. ${ }^{12}$ Because of its high accuracy, high efficiency, and easy implementation, the GS method has already been the most popular method in two-frame PSI and is used in many applications. ${ }^{13-15}$ The Self-Tuning (ST) method $^{16}$ uses a parameter-tuned "complex quadrature filter" to filter the interferograms in the Fourier domain. In this way, the frequency component that only includes the phase information is selected. The accuracy of the ST method decreases when the phase step moves away from $\pi / 2$ $\mathrm{rad}^{12}$ and is generally time-consuming as well. Moreover, its performance is very unstable. ${ }^{17}$ The extreme value of the interference (EVI) method retrieves the phase step between the two interferograms through the data at the extrema of interferograms. ${ }^{18}$ This method requires that the interferograms should be normalized in advance and the positions of extrema depend on noise and modulation variations. In holographic interferometry, ${ }^{19,20}$ the object wave intensity and reference wave intensity are incorporated into the retrieval of phase step along with the intensity statistical features of the two-frame interferograms. In Ref. 21, the phase reconstruction is based on the differential evolution (DE) algorithm to search for the optimal phase Zernike coefficients which fit with the two-frame interferograms best. This method also needs to normalize the interferograms first and is extremely timeconsuming because of the complicate DE algorithm. In Ref. 22, the unknown phase step is estimated directly by solving a quartic polynomial equation, and very fast and accurate phase extraction is realized. However, it also requires that the interferograms should have more than one fringe.

In this letter, we report an elegant phase reconstruction method for random two-frame PSI, which is much different from the frameworks of existing methods. In the proposed approach, the phase of random two-frame PSI can be accurately reconstructed using the phase step value which minimizes the coefficient of variation $(\mathrm{CV})$ of the modulation term. To begin with, the two-frame phase-shifting interferograms can be modeled as

$$
\begin{aligned}
& I_{1}(x, y)=A(x, y)+B(x, y) \cos [\phi(x, y)], \\
& I_{2}(x, y)=A(x, y)+B(x, y) \cos [\phi(x, y)+\alpha],
\end{aligned}
$$

where $A(x, y)$ denotes the DC background, $B(x, y)$ is called the modulation term of the interferogram, $\phi(x, y)$ is the phase map to be reconstructed, and $\alpha$ is the unknown phase step between the two interferograms. In connection with the practical interferograms, $A(x, y)$ $=I_{a}(x, y)+I_{b}(x, y)$ and $B(x, y)=2 \sqrt{I_{a}(x, y) I_{b}(x, y)}$, with $I_{a}$ and $I_{b}$ being the optical intensities of the two illuminated interference beams, which are slowly varying generally. In all of the existing two-frame reconstruction methods, the DC term $A(x, y)$ needs to be filtered out first, which can be readily done by many common methods such as simple Gaussian filtering, Hilbert-Huang adaptive filtering, ${ }^{15,23}$ and variational image decomposition ${ }^{24}$ because of its slowly varying essence as illustrated above. So we also start from the DC-removed interferograms here. Then, the modulation term $B(x, y)$ is expressed as a function of $\alpha$,

$$
B=\sqrt{I_{1}^{2}+\left[I_{1} / \tan (\alpha)-I_{2} / \sin (\alpha)\right]^{2}},
$$

where the coordinate dependence is ignored for simplicity. The CV of the modulation term is defined as

$$
C_{V}(B)=\operatorname{std}(B) / \operatorname{mean}(B),
$$

where mean $(\cdot)$ represents the mean value and $\operatorname{std}(\cdot)$ the standard deviation. From Eq. (3), the CV is actually defined as the standard deviation of the modulation term relative to its mean value. The normalization to the mean is very important in the CV definition because it makes this quantity independent of the scales of different measurements. Due to this characteristic of $\mathrm{CV}$, it can be used as a loyal indicator to evaluate the overall variation of the modulation term in different situations. The proposed CV minimization (CVM) method locates the phase step $\alpha$ at the position where the $\mathrm{CV}$ of the retrieved modulation term is minimized, i.e.,

$$
\alpha=\arg \min C_{V}(B) \text {. }
$$

After obtaining the phase step based on Eq. (4), the corresponding phase map is reconstructed by

$$
\phi=\tan ^{-1}\left\{\left[I_{1} \cos (\alpha)-I_{2}\right] /\left[I_{1} \sin (\alpha)\right]\right\} .
$$

To elaborate on the idea of the CVM method, Figs. 1(a) and 1(b) show two simulated interferograms with a phase step of $1.5 \mathrm{rad}$. In the simulation, additive white Gaussian noise is added into the interferograms with a signal-to-noise ratio (SNR) of $35 \mathrm{~dB}$ [by MATLAB function $\operatorname{awgn}(x, S N R)]$. The corresponding wrapped theoretical phase map is presented in Fig. 1(c). To simulate the possible uneven illumination in real interferometry, we use two Gaussian functions to generate the modulation term, as given in Fig. 1(d). The background term is set to zero in this simulation. Figure 1 (e) shows the CVs of the retrieved modulation terms [Eqs. (2) and (3)] using different phase steps based on the interferograms in Figs. 1(a) and 1(b). It can be seen that the true phase step $(1.5 \mathrm{rad})$ is just around the minimum point of the CV curve. The underlying reason for this phenomenon is easy to understand: the retrieved modulation term would have clear abnormal oscillation and larger overall variation at wrong phase steps (thus larger CVs), which do not fit with the physical essence of the real interferograms. For further demonstration, the insets of Fig. 1(e) show the calculated modulation terms at phase steps of $0.5 \mathrm{rad}, 1.5 \mathrm{rad}$, and $2 \mathrm{rad}$, respectively. We can see that the modulation terms at $0.5 \mathrm{rad}$ and $2 \mathrm{rad}$ are obviously much more fluctuant than that at the true phase step of $1.5 \mathrm{rad}$. Moreover, the CVs constitute a smooth curve albeit considerable noise is added into interferograms, which is beneficial from the statistical essence of CV. Thus, it can be a very reliable metric for retrieving the optimal phase steps between the phaseshifting interferograms. In this way, the phase reconstruction is converted into a CV minimization problem with respect to the phase step. This minimization can be accelerated through many ready-made 

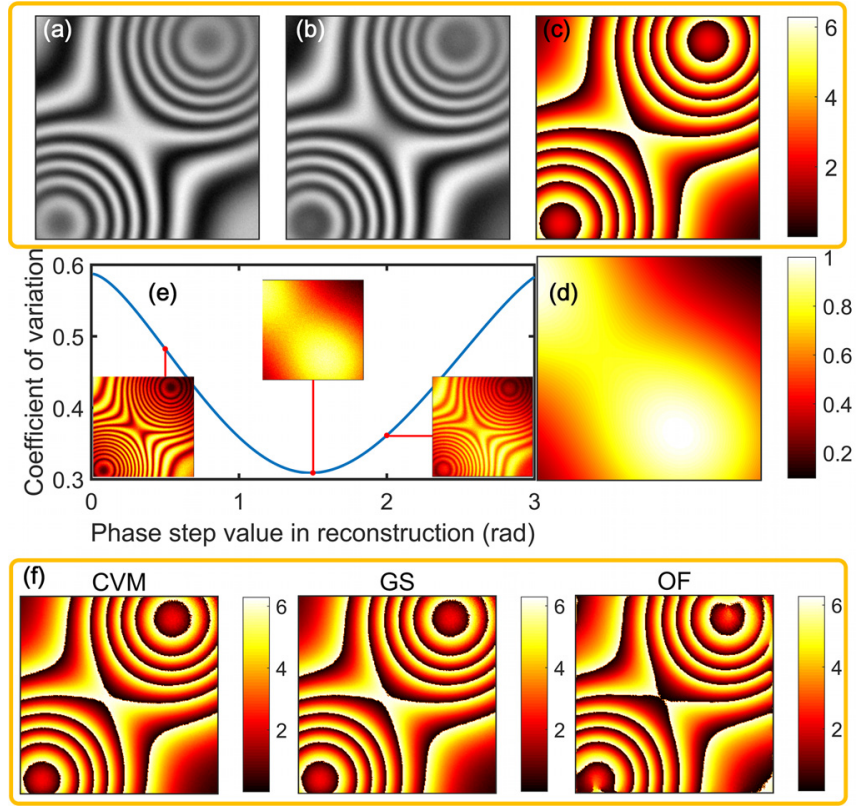

FIG. 1. Illustration of the proposed CVM method. (a) and (b) Two simulated interferograms with phase step of $1.5 \mathrm{rad}($ size $256 \times 256$ ). (c) Wrapped theoretical phase map (rad). (d) Simulated modulation term (a.u.). (e) Coefficients of variation (CVs) of the calculated modulation terms using different phase step values. The insets show the calculated modulation terms using phase steps of $0.5 \mathrm{rad}, 1.5 \mathrm{rad}$, and 2 rad, respectively. (f) Reconstructed wrapped phases by the proposed CVM, $\mathrm{GS}$, and OF methods (rad).

one-dimensional search algorithms. Here, we recommend the classical Fibonacci search algorithm ${ }^{25}$ which, according to our practices, can find the accurate solution within 15 search steps by uniformly setting the search lower limit and upper limit to 0 and $\pi$ rad, respectively. Moreover, the Fibonacci search is very easy to implement. Hence, we use this optimization technique as a tool to solve the CV minimization problem indicated by Eq. (4). For the phase-shifting interferograms in Figs. 1(a) and 1(b), the phase step is located at 1.49 rad through the Fibonacci search with a time of $0.033 \mathrm{~s}$ (see Table I). The reconstructed wrapped phase map from Eq. (5) using the retrieved phase step is shown in Fig. 1(f), where the results from the popular $\mathrm{GS}^{12}$ and $\mathrm{OF}^{11}$ methods are also presented for comparison. We quantify the phase reconstruction error by taking the difference between the reconstructed wrapped phase and the theoretical wrapped phase and calculating the root mean square (RMS) of the phase difference, which is listed in Table I. From Fig. 1(f) and Table I, it validates that the proposed CVM method can accomplish the phase reconstruction for two-frame PSI accurately and efficiently, even compared with these state-of-the-art methods.

As mentioned previously, many existing methods for random two-frame PSI require that the number of fringes in the interferograms should be more than one because some critical approximations in these methods require this precondition. In our CVM method, it seems that there is no limitation to this condition in principle. To verify this point, Figs. 2(a)-2(c) show two interferograms and their theoretical phase map, respectively, where the phase variation of the whole map is less than $2 \pi \mathrm{rad}$ (i.e., less than one fringe in interferograms).
TABLE I. Root-Mean-Square (RMS) errors between the reference wrapped phases and the obtained wrapped phases via CVM, GS, and OF methods and processing times. All the data are verified on the laptop with Intel Core i7-7500U processor and MATLAB R2017b.

\begin{tabular}{lcccc}
\hline \hline Figures & & CVM & GS & OF \\
\hline Figure 1 & RMS (rad) & 0.404 & 0.406 & 0.990 \\
& Time (s) & 0.033 & 0.010 & 0.358 \\
Figure 2 & RMS (rad) & 0.083 & 0.290 & 0.599 \\
& Time (s) & 0.022 & 0.006 & 0.192 \\
Figure 4(a) & RMS (rad) & 0.916 & 0.916 & 1.132 \\
& Time (s) & 0.064 & 0.022 & 0.624 \\
Figure 4(b) & RMS (rad) & 0.909 & 0.917 & 0.969 \\
& Time (s) & 0.371 & 0.133 & 3.070 \\
Figure 5 & RMS (rad) & 0.258 & 0.389 & 0.549 \\
& Time (s) & 0.025 & 0.007 & 0.655 \\
\hline \hline
\end{tabular}

The reconstructed phase maps as well as the corresponding phase reconstruction errors from the CVM, GS, and OF methods are presented in Figs. 2(d)-2(f), respectively. It can be found that the performance of the proposed CVM method is much superior to that of the
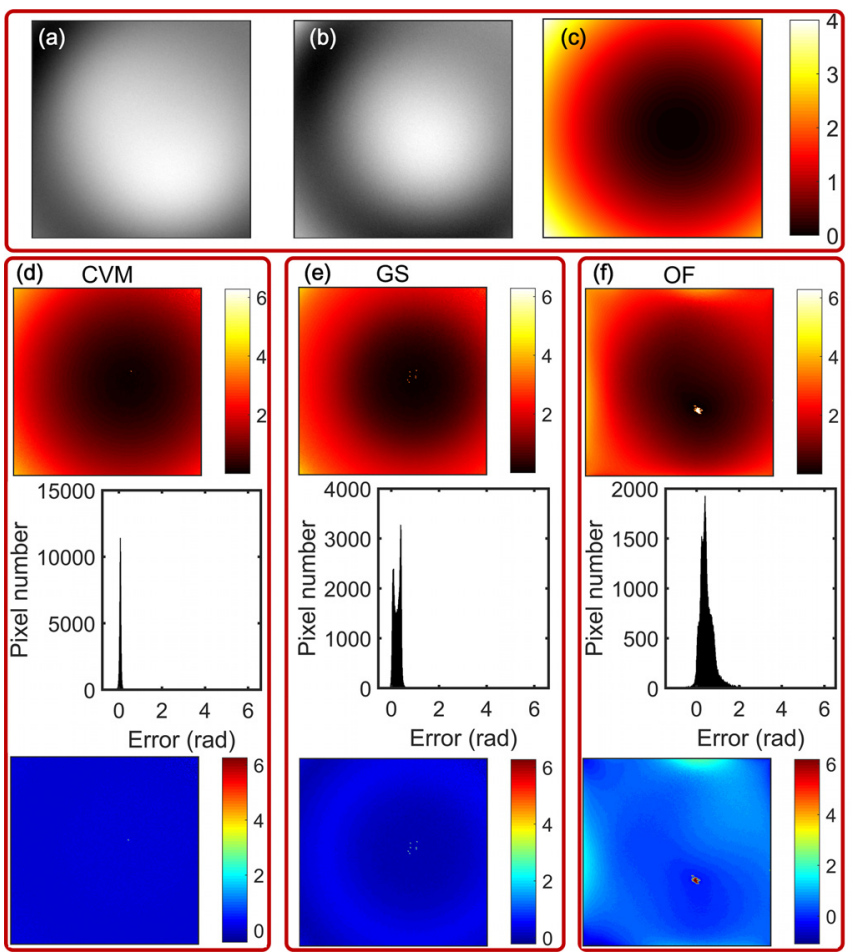

FIG. 2. Phase reconstruction for simulated interferograms with less than one fringe. (a)-(c) are the two interferograms (size $256 \times 256$ ) and the theoretical phase map (rad) in the simulation, respectively. The modulation term used here is similar to that in Fig. 1(d). (d) The reconstructed phase map (up, rad), the error histogram (middle) and the corresponding error map calculated as the difference between the reconstructed phase and the theoretical phase (down, rad) via the proposed CVM method. (e) and (f) Same as (d) except that the plots are for GS method and OF method, respectively. 
GS and OF methods in this case, in terms of the RMS errors summarized in Table I. Moreover, we see that the errors from the CVM method have natural Gaussian distribution with nearly zero mean, while those from GS and OF have obvious nonzero means and abnormal distributions, as shown in the histograms in Figs. 2(d)-2(f).

It is also interesting to examine the performance of the CVM method for two-frame PSI with different numbers of fringes in the interferograms and at different phase-shifting steps. To put the proposed method into a broader comparative context, we also added the ST method ${ }^{16}$ into the comparisons here. In Fig. 3(a), we show the phase reconstruction RMS errors for interferograms with different numbers of fringes. The employed interferograms are generated through setting the dynamic ranges of the simulated phase maps to $N \times 2 \pi \mathrm{rad}$, where $N$ is the number of fringes. From Fig. 3(a), we see that the accuracy of the CVM does not deteriorate with the decrease in the fringe number in interferograms, while that of the GS and OF methods degrades obviously when the fringe number is less than 1 , as expected in principle. The robustness of the CVM method for interferograms at different phase-shifting steps is also tested; the results are shown in Fig. 3(b). In this testing, the number of fringes is set to 1 such that the GS and OF methods can work normally. As can be seen, the CVM method performs well over the whole testing range of phase steps according to Fig. 3(b). Note that the retrieval errors for all of these two-frame methods become larger at the phase steps approaching the singular 0 and $\pi \mathrm{rad}$, which is inevitable for the two-frame reconstruction due to the limited information from interferograms at singular phase steps. The estimated phase steps as well as the
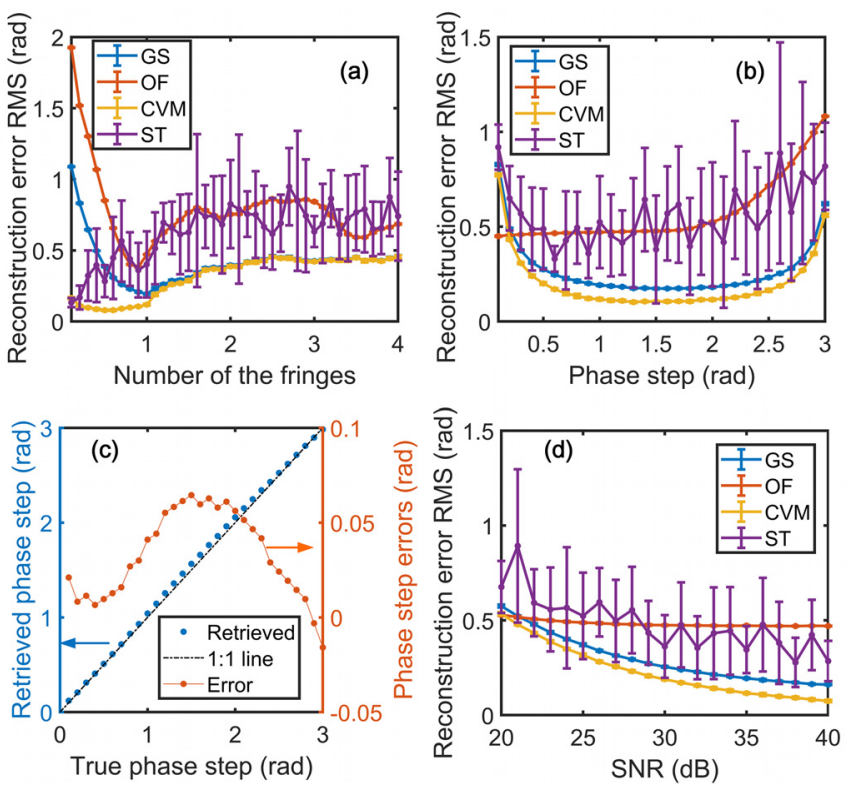

FIG. 3. Comprehensive comparisons on the phase reconstruction errors between different methods. (a) RMS errors for interferograms with different fringe numbers; (b) RMS errors for interferograms at different phase shift steps; (c) estimated phase steps as well as the estimation errors corresponding to the true ones in (b) via the CVM method; (d) RMS errors at different SNRs. The error bars in each figure are based on the statistical standard deviation of the RMS errors from repetitive phase reconstructions for 10 times with randomly changing noise in the interferograms. estimation errors corresponding to the true ones used in simulations of Fig. 3(b) are presented in Fig. 3(c) to verify the CVM method further. At last, we test the CVM method at different SNRs in Fig. 3(d), which indicates that it can perform very satisfactorily at different noise levels, compared with the current approaches. We note that the error bars in Fig. 3 are based on the statistical standard deviation of the RMS errors from repetitive phase reconstructions for 10 times with randomly changing noise in the interferograms. It demonstrates the good repeatability and stabilization of the proposed method as well as the popular GS and OF methods. From Fig. 3, it can be clearly seen that the accuracy of the ST method is generally much lower compared to that of other methods and, more noticeably, is very unstable, as also observed in Ref. 17.

Experimental interferograms are employed to corroborate the proposed CVM methods as well, as shown in Fig. 4. The interferograms in Figs. 4(a1) and 4(a2) are from interferometric testing for an aspheric surface. The wrapped reference phase map is obtained through the Advanced Iterative Algorithm (AIA) method $^{26}$ from 9 phase-shifting interferograms that contain the ones in Figs. 4(a1) and 4(a2), which is presented in Fig. 4(a3). After removing the backgrounds of the interferograms via simple Gaussian filtering, the CVM, GS, and OF methods are carried out to demodulate the phase
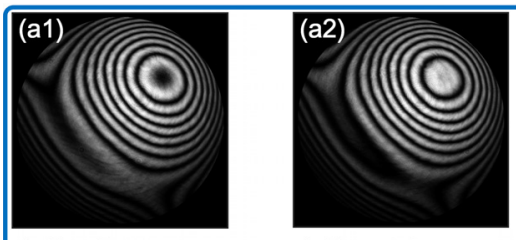

(a4) CVM

(a5) GS
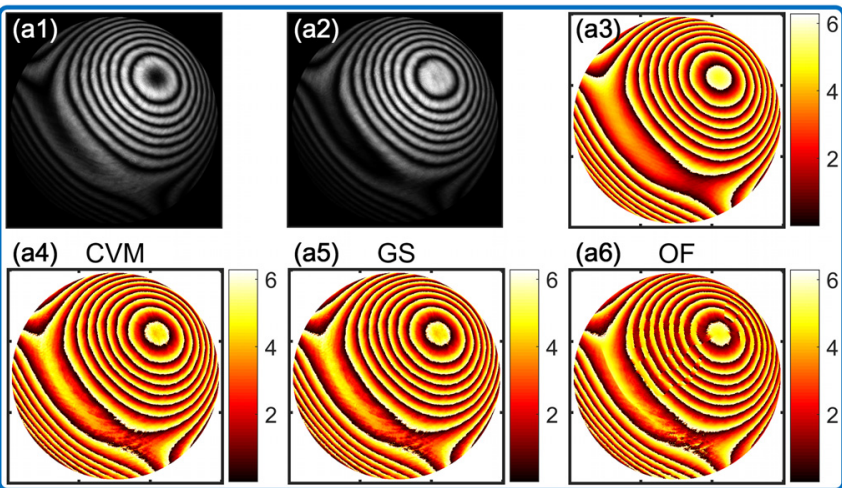

(a6) OF
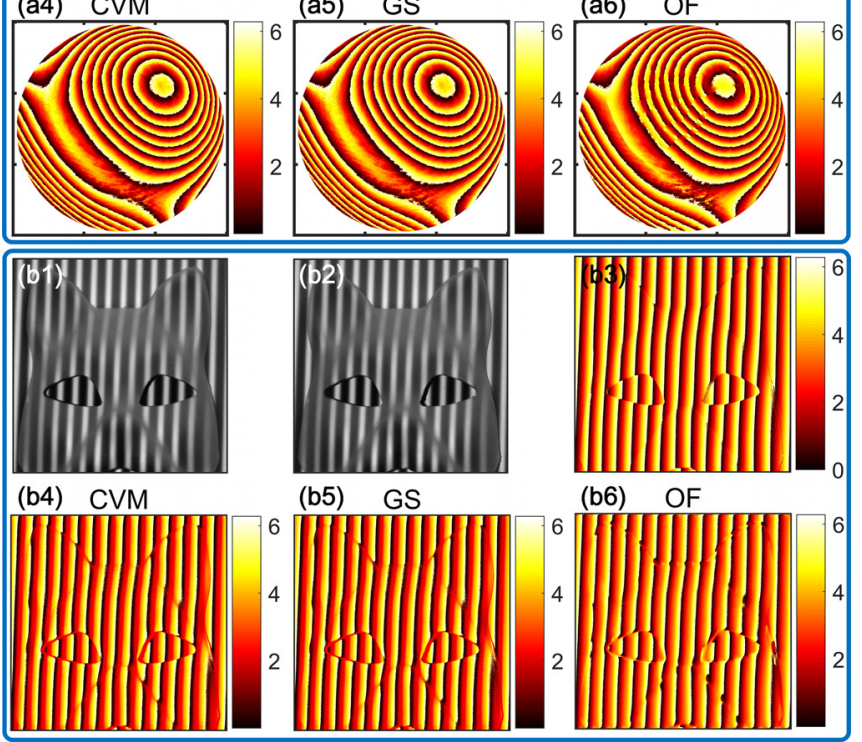

FIG. 4. Experimental phase reconstructions. (a1), (a2) and (b1), (b2) are two sets of phase-shifting interferograms [size $602 \times 602$ for set (a), and size $1177 \times 1609$ for set (b)]; (a3) and (b3) are the wrapped reference phase maps (rad) [(a3) is from the AIA method using 9 interferograms and (b3) is from standard 4-step phase shifting algorithm]; (a4)-(a6) and (b4)-(b6) are the reconstructed wrapped phase maps by CVM, GS, and OF methods for the two sets of interferograms (rad). 


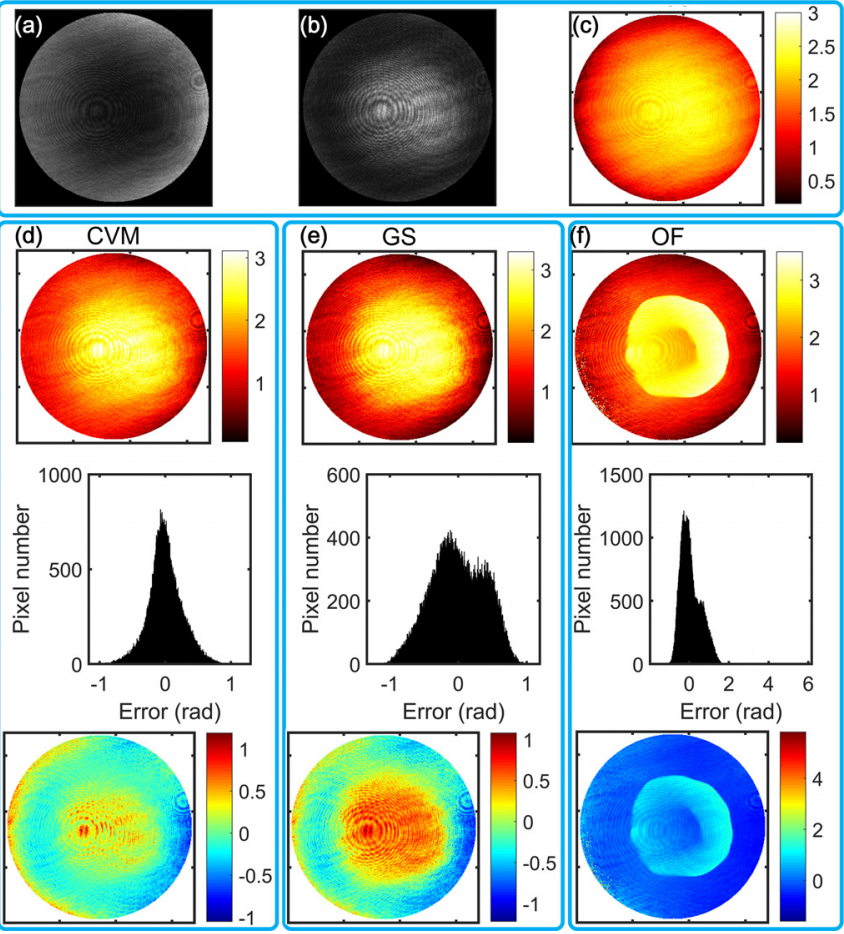

FIG. 5. Phase reconstruction for experimental interferograms with less than one fringe. (a)-(c) are the two experimental interferograms (size $341 \times 340)$ and the reference phase map (rad) from 9-frame AIA method, respectively. (d) The reconstructed phase map (up, rad), the error histogram (middle) and the corresponding error map calculated as the difference between the reconstructed phase and the reference phase (down, rad) via the proposed CVM method. (e), (f) Same as (d) except that the plots are for GS method and OF method, respectively.

map, and the results are shown in Figs. 4(a4)-4(a6). All of the three methods accomplish the phase reconstructions well in this case. From Table I, we see that the CVM and GS methods have similar accuracy for demodulating these experimental interferograms with many fringes, as also verified in Fig. 3(a). As an illustration of phase reconstruction for more general phase maps, we also show an example using two-frame interferograms from the fringe projection profilometry, ${ }^{3}$ as presented in Figs. 4(b1) and 4(b2). In this example, a discontinuous catlike mask is used as the testing target. The wrapped reference phase map by the standard 4-step phase shifting algorithm is given in Fig. 4(b3). From the reconstructed wrapped phase maps in Figs. 4(b4)-4(b6) and the RMS errors in Table I, one can see that the proposed CVM also works very well in this case and has better accuracy than the GS and OF methods.

We also use experimental interferograms with less than one fringe, as shown in Figs. 5(a) and 5(b), to validate the CVM method. The reference phase map from the AIA method with 9-frame interferograms is given in Fig. 5(c). The phase reconstruction results as well as the corresponding phase reconstruction errors through the CVM, GS, and OF methods are presented in Figs. 5(d)-5(f), respectively. Intuitively, the shapes of phase maps from the CVM and GS methods conform to the reference phase well, while that from the OF manifests conspicuous artifacts. Similar to the simulation results in Fig. 2, the error histograms show that the demodulation errors of the CVM method are much closer to Gaussian distribution with zero mean. According to Table I, it is obvious that the CVM method has much better accuracy than the GS and OF methods for demodulating the interferograms with fewer fringes, which is also consistent with the simulation results.

In summary, an accurate and efficient phase reconstruction method for random two-frame PSI, called CV minimization (CVM), is proposed in this work. Both numerical simulations and experimental data demonstrate that the CVM method can realize accurate phase reconstruction with high efficiency for random two-frame PSI, even compared with the most popular methods in this realm. In addition, the application restriction concerning the number of fringes in interferograms is not necessary, in contrast to those commonly used methods. We anticipate the extensive applications of the CVM method in random two-frame PSI in the future.

We would like to thank Dr. David Garrett for proofreading this manuscript. The authors acknowledge financial support from the National Key Research and Development Program of China (No. 2016YFC0200700) and National Natural Science Foundation of China (NSFC) (No. 61475141).

\section{REFERENCES}

${ }^{1}$ D. Malacara, Optical Shop Testing (Wiley, 2007).

${ }^{2}$ X. Zhou, R. Guo, W. Zhu, D. Zheng, and L. Chen, Appl. Opt. 56, 8040 (2017).

${ }^{3}$ S. Zhang, Opt. Lasers Eng. 48, 149 (2010).

${ }^{4}$ J. Yao, Y. Tang, and J. Chen, Opt. Lett. 41, 717 (2016).

${ }^{5}$ D. G. A. Ibrahim and T. Yasui, Appl. Phys. Lett. 112, 171101 (2018).

${ }^{6}$ K. Freischlad and C. L. Koliopoulos, J. Opt. Soc. Am. A 7, 542 (1990).

${ }^{7}$ D. S. Mehta and V. Srivastava, Appl. Phys. Lett. 101, 203701 (2012).

${ }^{8}$ M. Servin, J. L. Marroquin, and F. J. Cuevas, Appl. Opt. 36, 4540 (1997).

${ }^{9}$ K. G. Larkin, D. J. Bone, and M. A. Oldfield, J. Opt. Soc. Am. A 18, 1862 (2001).

${ }^{10}$ K. G. Larkin, J. Opt. Soc. Am. A 18, 1871 (2001).

${ }^{11}$ J. Vargas, J. A. Quiroga, C. O. S. Sorzano, J. C. Estrada, and J. M. Carazo, Opt. Lett. 36, 3485 (2011).

${ }^{12}$ J. Vargas, J. A. Quiroga, C. O. S. Sorzano, J. C. Estrada, and J. M. Carazo, Opt. Lett. 37, 443 (2012).

${ }^{13}$ Y. Lu, R. Li, and R. Lu, Appl. Opt. 55, 6866 (2016).

${ }^{14}$ W. Zhang, X. Lu, L. Fei, H. Zhao, H. Wang, and L. Zhong, Opt. Lett. 39, 5375 (2014).

${ }^{15}$ M. Trusiak and K. Patorski, Opt. Express 23, 4672 (2015).

${ }^{16}$ J. Vargas, J. A. Quiroga, T. Belenguer, M. Servín, and J. C. Estrada, Opt. Express 19, 638 (2011).

${ }^{17}$ D. Saide, M. Trusiak, and K. Patorski, Appl. Opt. 56, 5489 (2017).

${ }^{18}$ J. Deng, H. Wang, F. Zhang, D. Zhang, L. Zhong, and X. Lu, Opt. Lett. 37, 4669 (2012).

${ }^{19}$ X. F. Xu, L. Z. Cai, Y. R. Wang, X. F. Meng, W. J. Sun, H. Zhang, X. C. Cheng, G. Y. Dong, and X. X. Shen, Opt. Lett. 33, 776 (2008).

${ }^{20}$ X. F. Xu, L. Z. Cai, Y. R. Wang, X. L. Yang, X. F. Meng, G. Y. Dong, X. X. Shen, and H. Zhang, Appl. Phys. Lett. 90, 121124 (2007).

${ }^{21}$ C. Tian and S. Liu, Opt. Express 25, 21673 (2017).

${ }^{22}$ Z. Cheng and D. Liu, Opt. Lett. 43, 3033 (2018).

${ }^{23}$ X. Zhou, H. Zhao, and T. Jiang, Opt. Lett. 34, 2033 (2009).

${ }^{24} \mathrm{X}$. Zhu, Z. Chen, and C. Tang, Opt. Lett. 38, 275 (2013).

${ }^{25}$ E. K. Chong and S. H. Zak, An Introduction to Optimization (John Wiley \& Sons, 2013).

${ }^{26} \mathrm{Z}$. Wang and B. Han, Opt. Lett. 29, 1671 (2004). 